\title{
Histerectomia Totalmente Laparoscópica: Análise Retrospetiva de 262 Casos
}

\author{
Total Laparoscopic Hysterectomy: Retrospective Analysis of 262 Cases
}

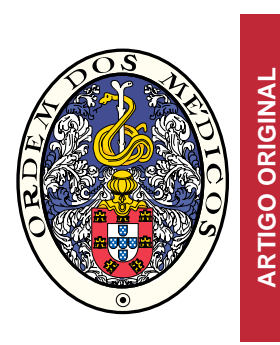

\author{
Cristina NOGUEIRA-SILVA ${ }^{1,2}$, Samuel SANTOS-RIBEIRO ${ }^{3,4}$, Sónia BARATA ${ }^{3,4}$, Conceição ALHO ${ }^{3,4}$, Filipa OSÓRIO $^{3,4}$, \\ Carlos CALHAZ-JORGE ${ }^{3,4}$ \\ Acta Med Port 2014 Jan-Feb;27(1):73-81
}

\section{RESUMO}

Introdução: A histerectomia é a cirurgia ginecológica major mais frequentemente realizada nos países desenvolvidos, considerando-se três principais vias de abordagem: vaginal, abdominal e laparoscópica. Apesar de múltiplas vantagens, a histerectomia totalmente laparoscópica tem-se associado a controvérsia relativamente à taxa de complicações.

Objectivos: Análise da nossa casuística de histerectomia totalmente laparoscópica e avaliação da taxa de complicações.

Material e Métodos: Análise retrospetiva dos processos clínicos das doentes submetidas a histerectomia totalmente laparoscópica no nosso departamento, pela mesma equipa cirúrgica, entre abril de 2009 e março de $2013(n=262)$.

Resultados: As doentes tinham em média 48,9 9 anos e 49,2\% tinha antecedentes de cirurgia abdomino-pélvica. O índice de massa corporal médio era $26,5 \pm 4,5 \mathrm{~kg} / \mathrm{m}^{2}$, sendo que $42 \%$ eram obesas ou tinham excesso de peso. O tempo operatório médio para realização da histerectomia totalmente laparoscópica foi $77,7 \pm 27,5$ minutos, diminuindo significativamente com o aumento da experiência da equipa cirúrgica. O peso médio da peça operatória foi $241 \pm 168,4 \mathrm{~g}$ e a duração média do internamento após a cirurgia foi $1,49 \pm$ 0,9 dias. A diferença entre a hemoglobina pré e pós-operatória foi $1,5 \pm 0,8 \mathrm{~g} / \mathrm{dL}$. A morbilidade major foi $1,5 \%(n=4)$ e a minor $11,5 \%$ $(n=30)$. Salienta-se um caso de conversão para laparotomia e dois casos de deiscência da cúpula vaginal. Não ocorreu nenhuma lesão urinária ou gastrointestinal grave.

Conclusões: Esta série demonstra que, se realizada por uma equipa cirúrgica adequadamente treinada, a histerectomia totalmente laparoscópica é segura e associada a baixa taxa de complicações.

Palavras-chave: Complicações Pós-Operatórias; Histerectomia; Laparoscopia; Portugal.

\section{ABSTRACT}

Introduction: Hysterectomy is one of the most common gynecological procedures and may be performed either by vaginal approach, laparotomy or laparoscopy. Although total laparoscopic hysterectomy has multiple advantages, conflicting major complication rates have been previously reported.

Objectives: To describe our experience performing TLH and to evaluate complication rates.

Material and Methods: A retrospective observational study of all total laparoscopic hysterectomy performed in our department, by the same surgical team, between April 2009 and March $2013(n=262)$, was conducted. Medical records were reviewed for patient characteristics, operating time, uterine weight, post-operative hemoglobin variation, length of hospital stay, and intra and postoperative complications.

Results: Patient average age was $48.9 \pm 9.0$ years and $49.2 \%$ had previous abdominopelvic surgery. The average body mass index was $26.5 \pm 4.5 \mathrm{~kg} / \mathrm{m}^{2}$ and $42 \%$ of women were either overweight or obese. The mean operating time during the total study period was $77.7 \pm 27.5$ minutes, but it decreased significantly as the surgical team's training increased. Average uterine weight was $241.0 \pm 168.4 \mathrm{~g}$ and average hospital stay was $1.49 \pm 0.9$ days. The mean postoperative hemoglobin variation was $-1.5 \pm 0.8 \mathrm{~g} / \mathrm{dL}$. The major and minor complication rates were $1.5 \%(n=4)$ and $11.5 \%(n=30)$, respectively. One procedure was converted to laparotomy and two women had a vaginal vault dehiscence. No important urinary tract or bowel injuries occurred.

Conclusions: This study demonstrates that, in experienced hands, total laparoscopic hysterectomy is safe and with low complications rates. Keywords: Hysterectomy; Laparoscopy; Postoperative Complications; Portugal.

\section{INTRODUÇÃO}

A histerectomia é a cirurgia ginecológica major mais frequentemente realizada nos países desenvolvidos. ${ }^{1,2}$ Nos Estados Unidos estima-se atualmente uma incidência de 1:461 mulheres, o que corresponde a cerca de 600000 histerectomias por ano..$^{3-6}$ Por sua vez, no Canadá, em 2008-2009, realizaram-se 338 histerectomias por 100000 mulheres com idade superior a 20 anos. $^{2}$

Desde a descrição por Reich et al da primeira histerectomia laparoscópica, em 1989, que se consideram três principais abordagens para a histerectomia: a via vaginal, a via abdominal e a via laparoscópica. ${ }^{7}$ Diversos estudos têm analisado as vantagens, desvantagens e taxa de complicações associadas a cada tipo de histerectomia. .,8-10 Numa $^{4}$ fase inicial a histerectomia laparoscópica foi apresentada como associando-se a elevada taxa de complicações, nomeadamente lesões do trato urinário. ${ }^{11,12}$ Nesse sentido defendeu-se que a histerectomia vaginal deveria ser a abordagem de primeira linha, sempre que possível, dadas

\footnotetext{
1. Serviço de Ginecologia e Obstetrícia. Hospital de Braga. Braga. Portugal.
}

2. Instituto de Investigação em Ciências da Vida e da Saúde. Escola de Ciências da Saúde. Universidade do Minho. Braga. Portugal.

3. Departamento de Obstetrícia, Ginecologia e Medicina da Reprodução. Hospital de Santa Maria. Centro Hospitalar Lisboa Norte. Lisboa. Portugal.

4. Faculdade de Medicina. Universidade de Lisboa. Lisboa. Portugal.

Recebido: 30 de Maio de 2013 - Aceite: 09 de Outubro de 2013 | Copyright $\odot$ Ordem dos Médicos 2014 
as suas vantagens documentadas (menor duração da intervenção cirúrgica e do internamento, menos dor pós-operatória, regresso precoce à atividade profissional) e baixa taxa de complicações. A histerectomia laparoscópica deveria, assim, ser realizada se a via vaginal não fosse exequível, a fim de se evitar uma histerectomia abdominal. ${ }^{6,8}$ Contudo, estudos mais recentes têm demonstrado que a histerectomia laparoscópica é segura e se associa a baixa taxa de complicações. ${ }^{13,14}$ Donnez et al, após análise de 3190 histerectomias laparoscópicas, demonstraram que a via laparoscópica apresenta múltiplas vantagens comparativamente com a via vaginal e abdominal, com morbilidade similar. ${ }^{9}$ Apesar da controvérsia, os diferentes autores são consensuais a defender a importância da curva de aprendizagem e experiência das equipas cirúrgicas nos resultados publicados, bem como a existência de indicações espe-

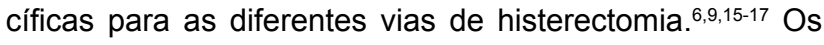
ginecologistas deverão, assim, desenvolver competências adequadas e treino em todas as abordagens de histerectomia, a fim de que a escolha da via de abordagem seja realizada de forma individualizada, tendo em consideração a indicação cirúrgica, as caraterísticas da doente e a sua vontade. ${ }^{17}$ Contudo, existem evidências que a seleção da via da histerectomia se baseia mais na preferência e experiência do cirurgião do que na literatura. ${ }^{18}$

Em Portugal, a experiência com histerectomia laparoscópica é ainda reduzida. Um estudo promovido pelo Colégio de Especialidade de Ginecologia e Obstetrícia da Ordem dos Médicos revelou que, em 2011, em 34 hospitais nacionais, apenas $13 \%$ das histerectomias foram realizadas por esta via. A inexistência de profissionais com treino e experiência suficientes foi apontada como o principal obstáculo ao desenvolvimento da cirurgia laparoscópica. ${ }^{19}$

Neste trabalho apresentamos a análise de 262 histerectomias totalmente laparoscópicas (HTL) realizadas pela mesma equipa cirúrgica.

\section{MATERIAL E MÉTODOS}

Trata-se de um estudo descritivo, observacional e transversal, com análise retrospetiva dos processos clínicos de todas as doentes submetidas a HTL, pela mesma equipa cirúrgica e de acordo com a mesma técnica, entre 1 de abril de 2009 e 31 de março de 2013.

As doentes foram observadas em consulta 1, 6 e 12 meses após a intervenção (exceto as cirurgias realizadas posteriormente a 31 de março de 2012, cujo seguimento

Tabela 1 - Complicações intra e pós-operatórias major e minor

\title{
Complicações Major
}

\author{
Lesão ureteral \\ Lesão vesical \\ Perfuração intestinal \\ Hemorragia com necessidade de transfusão sanguínea \\ Hematoma da cúpula com necessidade de drenagem cirúrgica ou transfusão sanguínea \\ Complicações anestésicas major \\ Conversão para laparotomia \\ Deiscência da cúpula vaginal \\ Embolia pulmonar
}

\section{Complicações Minor}

Hemorragia* sem necessidade de transfusão sanguínea

Hematoma (com drenagem espontânea)

Lesão iatrogénica da serosa intestinal

Infeção (urinária, da cicatriz, pélvica ou outra) ou febre (temperatura $>38^{\circ} \mathrm{C}$ nos primeiros 7 dias)

Trombose venosa profunda

Problemas anestésicos minor

Incontinência urinária

* Hemorragia definida pela avaliação subjetiva e, simultaneamente, confirmada pela diminuição da hemoglobina $\geq 3,5 \mathrm{~g} / \mathrm{dL}$ 
ainda não concluiu os 12 meses), com registo das complicações ocorridas e sintomatologia associada. Verificou-se uma taxa de falta à consulta de $1,1 \%(n=3)$ no primeiro mês, $7,3 \%(n=19)$ aos seis meses e 14,1\% $(n=37)$ aos 12 meses.

Para caracterização da população foram analisados os seguintes parâmetros: idade; índice de massa corporal (IMC); idade da menarca e menopausa; utilização de terapêutica hormonal; paridade; antecedentes médicos e cirúrgicos. Relativamente ao procedimento cirúrgico realizado foram avaliados: indicação cirúrgica principal; procedimentos cirúrgicos acessórios; tempo operatório; tipo de energia utilizada; utilização de trocar epigástrico; necessidade de morcelação da peça; peso da peça cirúrgica; duração do internamento após a cirurgia; diagnóstico anatomo-patológico; variação da hemoglobina e hematócrito no pré e pós-operatório; complicações major e minor intra-operatórias e pós-operatórias (ocorridas até 12 meses após a cirurgia), utilizando critérios de séries publicadas anteriormente (Tabela 1$) \cdot 9,11$

\section{Procedimento cirúrgico}

Após esclarecimento adequado, o consentimento informado foi assinado por todas as doentes previamente à cirurgia. As doentes foram internadas no dia anterior à intervenção cirúrgica, tendo realizado colheita de sangue para hemograma pré-operatório e preparação intestinal. Foi repetido hemograma no primeiro dia de pós-operatório.

Todas as cirurgias foram realizadas sob anestesia geral e com algaliação contínua com sonda de Foley. Foi realizada antibioticoprofilaxia venosa com $2 \mathrm{~g}$ de cefoxitina, em dose única.

A abordagem cirúrgica foi realizada com quatro incisões cutâneas, incluindo sempre o umbigo, habitualmente: um trocar umbilical de $10 \mathrm{~mm}$ para a óptica, dois trocares laterais e um trocar supra-púbico de $5 \mathrm{~mm}$ para os instrumentos cirúrgicos. Em algumas situações foi utilizado um trocar epigástrico de $10 \mathrm{~mm}$ em substituição do trocar supra-púbico.

Após criação do campo cirúrgico e previamente à HTL, foi realizada sempre uma laparoscopia diagnóstica com identificação do trajeto dos ureteres, seguido, quando necessário, pela lise de aderências ou tratamento de lesões de endometriose. Todas as HTL foram realizadas com o manipulador uterino de Clermont-Ferrand. Procedeu-se às HTL segundo a técnica de Clermont-Ferrand, começando pela laqueação do ligamento redondo esquerdo; disseção do folheto anterior do ligamento largo até ao nível da prega vesico-uterina; abertura de janela no folheto posterior do ligamento largo; laqueação do ligamento útero-ovárico ou ligamento infundíbulo-pélvico (se conservação ou ablação anexial, respetivamente); repetição dos mesmos passos para o lado direito; dissecção do espaço vesico-uterino com rebatimento da bexiga; laqueação bilateral das artérias uterinas, ligamentos cardinais e útero-sagrados; realização de colpotomia circular; extração da peça por via vaginal (sem ou com necessidade de morcelação); encerramento da cúpula vaginal por via laparoscópica, com suspensão dos li- gamentos útero-sagrados; revisão da hemostase, lavagem da cavidade e confirmação do peristaltismo dos ureteres. $O$ tempo operatório para realização da HTL foi considerado desde o início da laqueação do ligamento redondo esquerdo até à conclusão do encerramento da cúpula vaginal.

\section{Análise estatística}

Os dados foram introduzidos numa base de dados construída em FileMaker Pro 12 Advanced $^{\circledR}$. Na análise estatística foi utilizado o programa SigmaStat ${ }^{\circledR}$ (versão 3.5). Procedeu-se a uma análise descritiva, com medidas de tendência central e medidas de dispersão, tendo em conta as variáveis em estudo. Os resultados referentes a variáveis quantitativas são apresentados como média \pm desviopadrão. Recorreu-se ao teste Kolmogorov-Smirnov para análise da normalidade e teste qui-quadrado e KruskalWallis para amostras independentes. O teste de Dunn foi utilizado para comparações múltiplas emparelhadas. Admitiu-se significância estatística para valores de $p<0,05$.

\section{RESULTADOS}

Em quatro anos foram realizadas, pela mesma equipa cirúrgica, 549 histerectomias. Destas $47,7 \%(n=262)$ foram HTL, $36,4 \%(n=200)$ foram histerectomias abdominais e $15,9 \%(n=87)$ foram histerectomias vaginais. Como se pode observar na Fig. 1, o número de HTL aumentou ao longo do período de análise enquanto as histerectomias abdominais e vaginais diminuíram. Na verdade, a taxa de HTL aumentou de $32,9 \%(n=47)$ no primeiro ano para $53,1 \%(n=68)$ no quarto ano $(p<0,001)$.

A idade média das 262 mulheres submetidas a HTL foi $48,9 \pm 9$ anos (mínimo 21; máximo 84). Os antecedentes ginecológicos e obstétricos, médicos e cirúrgicos de relevo são apresentados na Tabela 2. De referir que 15,6\% $(n=41)$ das mulheres eram nulíparas e 23,7\% $(n=62)$ eram pós-menopáusicas, tendo $17,7 \%(n=11)$ destas realizado terapêutica hormonal. Relativamente aos antecedentes médicos apenas 32,1\% $(n=84)$ eram saudáveis.

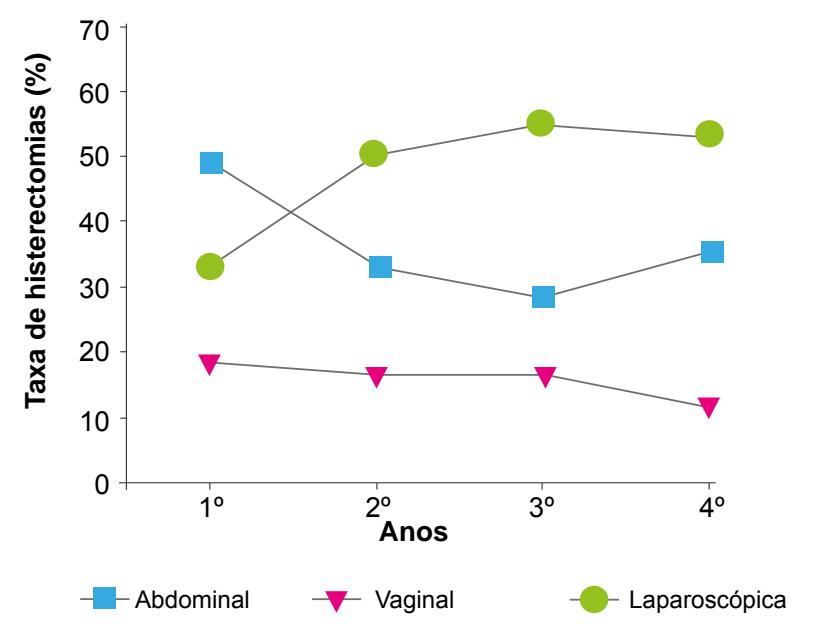

Figura 1 - Evolução da taxa de histerectomias abdominal, vaginal e HTL ao longo dos 4 anos do estudo 
Tabela 2 - Antecedentes ginecológicos e obstétricos, médicos e cirúrgicos das mulheres submetidas a HTL

\section{Antecedentes Ginecológicos e Obstétricos}

Menarca (média \pm DP; mín - máx)

$12,5 \pm 1,7(8-17)$

\section{Paridade}

Nulíparas, n (\%)

$41(15,6 \%)$

Multíparas, n (\%); média \pm DP

$221(84,4 \%) ; 1,9 \pm 0,8$

\section{Idade reprodutiva}

Pré-menopausa, n (\%)

$200(76,3 \%)$

Pós-menopausa, n (\%); média \pm DP

$62(23,7 \%) ; 50,8 \pm 3,6$

\begin{tabular}{|c|c|}
\hline \multicolumn{2}{|c|}{ Antecedentes Médicos } \\
\hline $\begin{array}{l}\text { Irrelevantes, } n(\%) \\
\text { Patologia associada, } \boldsymbol{n}(\%)\end{array}$ & $84(32,1 \%)$ \\
\hline Hipertensão arterial & $65(24,8 \%)$ \\
\hline Depressão & $35(13,4 \%)$ \\
\hline Dislipidemia & $27(10,3 \%)$ \\
\hline Patologia endocrinológica & $23(8,8 \%)$ \\
\hline Patologia oncológica & $17(6,5 \%)$ \\
\hline Diabetes mellitus tipo 2 & $12(4,6 \%)$ \\
\hline Patologia respiratória & $12(4,6 \%)$ \\
\hline Insuficiência venosa & $11(4,3 \%)$ \\
\hline Patologia cardíaca & $10(3,8 \%)$ \\
\hline Patologia hematológica & $10(3,8 \%)$ \\
\hline Outra & $26(9,9 \%)$ \\
\hline IMC (média \pm DP; mín - máx) & $26,5 \pm 4,5(19-43)$ \\
\hline Excesso de peso, $n(\%)$ & $71(27,1 \%)$ \\
\hline Obesidade, $n(\%)$ & $39(14,9 \%)$ \\
\hline \multicolumn{2}{|c|}{ Antecedentes Cirúrgicos } \\
\hline \multicolumn{2}{|c|}{ Cirurgia ginecológica via vaginal, $n$ (\%) } \\
\hline Histeroscopia & $47(17,9 \%)$ \\
\hline Ressectoscopia & $14(5,3 \%)$ \\
\hline Uroginecológica & $4(1,5 \%)$ \\
\hline Conização & $3(1,1 \%)$ \\
\hline \multicolumn{2}{|c|}{ Cirurgia ginecológica via abdominal, $n$ (\%) } \\
\hline Cesariana & $47(17,9 \%)$ \\
\hline Laparoscopia cirúrgica & $36(13,7 \%)$ \\
\hline Laparotomia & $27(10,3 \%)$ \\
\hline Laparoscopia diagnóstica & $3(1,1 \%)$ \\
\hline \multicolumn{2}{|c|}{ Cirurgia abdominal não ginecológica, $n$ (\%) } \\
\hline Apendicectomia & $31(11,8 \%)$ \\
\hline Colecistectomia & $20(7,6 \%)$ \\
\hline Outras & $4(1,5 \%)$ \\
\hline
\end{tabular}

IMC: índice de massa corporal; média \pm DP: média \pm desvio-padrão; mín - máx: valor mínimo e máximo; Menarca, idade reprodutiva: anos; IMC: $\mathrm{Kg} / \mathrm{m}^{2}$. 
Tabela 3 - Principal indicação cirúrgica das mulheres submetidas a HTL

\begin{tabular}{|c|c|c|}
\hline Indicação Cirúrgica & $n$ & $\%$ \\
\hline Útero miomatoso & 140 & 53,4 \\
\hline Polipose endometrial & 28 & 10,7 \\
\hline Patologia anexial & 27 & 10,3 \\
\hline Endometriose & 26 & 9,9 \\
\hline Hiperplasia endometrial & 14 & 5,3 \\
\hline Prolapso urogenital & 11 & 4,2 \\
\hline Adenomiose isolada & 7 & 2,7 \\
\hline Neoplasia do endométrio & 3 & 1,1 \\
\hline Neoplasia da mama & 2 & 0,8 \\
\hline Neoplasia do colo & 2 & 0,8 \\
\hline Doença hematológica & 1 & 0,4 \\
\hline Displasia do colo & 1 & 0,4 \\
\hline
\end{tabular}

Tabela 4 - Procedimentos cirúrgicos principais e acessórios realizados

Procedimento principal - HTL, $\boldsymbol{n}(\%)$

Com ablação anexial unilateral ou bilateral

$165(63,0 \%)$

Com conservação anexial

$84(32,1 \%)$

Com salpingectomia unilateral ou bilateral

$11(4,2 \%)$

Com ooforectomia unilateral

$2(0,8 \%)$

Procedimentos acessórios, $\boldsymbol{n}$ (\%)

Uroginecológicos, $n$ (\%)

Adesiólise

$46(54,1 \%)$

Colpoperineorrafia

$15(17,6 \%)$

Quistectomia unilateral ou bilateral

$10(11,8 \%)$

Excisão de nódulo de septo reto-vaginal

$9(10,6 \%)$

TVT-O

$8(9,4 \%)$

Cistoscopia

$7(8,2 \%)$

Salpingectomia unilateral

$6(7,1 \%)$

Excisão de nódulo vesical

Linfadenectomia pélvica

$1(1,2 \%)$

\section{Outros, $n$ (\%)}

Herniorrafia / hernioplastia

Shaving rectal

Omentectomia

Apendicectomia

Resseção intestinal 
A hipertensão arterial (24,8\%), a depressão $(13,4 \%)$ e a dislipidemia $(10,3 \%)$ foram as entidades nosológicas mais comummente associadas. O IMC médio foi $26,5 \pm 4,5 \mathrm{~kg} /$ $\mathrm{m}^{2}$, sendo que $14,9 \%$ das mulheres eram obesas $(n=39)$ e $27,1 \%(n=71)$ tinham excesso de peso, segundo os critérios da Organização Mundial de Saúde. ${ }^{20}$ No que diz respeito aos antecedentes cirúrgicos, $49,2 \%(n=129)$ das mulheres tinha pelo menos uma cirúrgica abdomino-pélvica prévia. Destas realça-se que $17,9 \%(n=47)$ tinha pelo menos uma cesariana anterior. Relativamente a outros antecedentes cirúrgicos abdominais salientam-se antecedentes de apendicectomia em $11,8 \%(n=31)$ e de colecistectomia em 7,6\% $(n=20)$ das doentes.

As principais indicações cirúrgicas foram: útero miomatoso (53,4\%; associado a sintomatologia, nomeadamente hemorragia uterina anormal refratária ao tratamento médico), polipose endometrial $(10,7 \%)$, patologia anexial $(10,3 \%)$, endometriose $(9,9 \%)$, hiperplasia endometrial $(5,3 \%)$ e prolapso urogenital $(4,2 \%$; prolapso uterino associado a útero globoso, miomatoso ou massa anexial simultânea) (Tabela 3).

Foi realizada HTL com ablação anexial unilateral ou bilateral em $63 \%$ ( $n=165)$ dos casos, com conservação bilateral de anexos em $32,1 \%(n=84)$, com salpingectomia unilateral ou bilateral em 4,2\% $(n=11)$ e ooforectomia unilateral em 0,8\% $(n=2)$ (Tabela 4). Em 32,4\% $(n=85)$ das doentes foram efetuados outros procedimentos cirúrgicos acessórios (Tabela 4), salientando-se adesiólise em 46 mulheres, ureterólise em cinco, exérese de nódulo de endometriose (do septo-retovaginal em nove casos, da bexiga e do intestino em um caso), e linfadenectomia pélvica em um caso (doente de 60 anos com adenocarcinoma do endométrio do tipo endometrióide com invasão superior a metade do miométrio).

O tempo operatório médio para realização da HTL foi $77,7 \pm 27,5$ minutos (mínimo 25; máximo 180). Como se pode observar na Fig. 2, o tempo cirúrgico médio diminuiu de forma estatisticamente significativa do primeiro $(87,4 \pm$ 26,8 minutos) para o quarto ano $(69,1 \pm 22,9$ minutos $)$ do

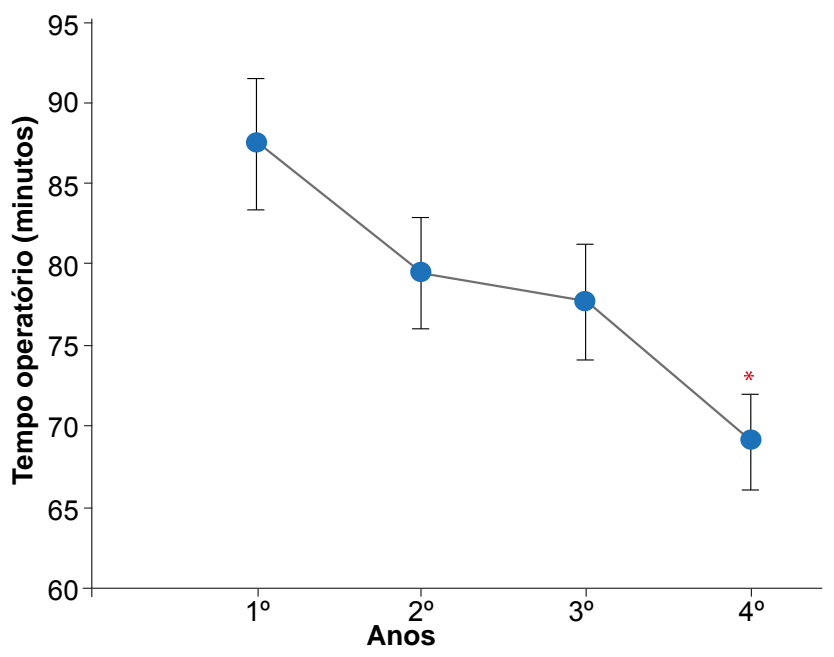

Figura 2 - Tempo operatório médio para realização de HTL ao longo dos 4 anos do estudo. $P<0,05:{ }^{*}$ vs. $1^{\circ}$ ano estudo $(p<0,05)$. Como sistema de energia foi utilizada a energia bipolar/monopolar em 93,5\% $(n=245)$ dos casos (energia bipolar para as laqueações, coagulação e disseção; energia monopolar apenas para a colpotomia circular). Por sua vez, foi utilizada energia ultrassónica (Ultracision ${ }^{\circledR}$ ) em 4,6\% $(n=12)$ dos casos, o sistema LigaSure ${ }^{\circledR}$ em $1,1 \%$ $(n=3)$ e o sistema Enseal ${ }^{\circledR}$ em $0,8 \%(n=2)$ dos casos. Tendo em consideração as dimensões uterinas, foi necessária a colocação de trocar epigástrico em 15,6\% $(n=41)$ dos casos. Foi necessária morcelação da peça operatória em $30,9 \%(n=81)$ das cirurgias, tendo sido realizada em $90,1 \%(n=73)$ dos casos por via vaginal. O peso médio da peça operatória foi $241 \pm 168,4 \mathrm{~g}$ (mínimo 34; máximo 1000). A duração média do internamento após a cirurgia foi $1,49 \pm 0,9$ dias, com $64,5 \%$ das doentes apresentando internamento de um dia (mínimo 1; máximo 8).

No que diz respeito aos achados anatomo-patológicos, os mais frequentes foram fibroleiomioma em $69,1 \%$ ( $n=$ $181)$ dos casos, adenomiose em $33,6 \%(n=88)$, pólipo endometrial em $17,2 \%(n=45)$, patologia do ovário em $14,5 \%$ $(n=38)$ e endometriose em $12,6 \%(n=33)$ dos casos.

A diferença média entre a hemoglobina pré e pós-operatória foi $1,5 \pm 0,8 \mathrm{~g} / \mathrm{dL}$, tendo a perda máxima sido $4,7 \mathrm{~g} /$ $\mathrm{dL}$. A diminuição da hemoglobina foi igual ou superior a 3,5 $\mathrm{g} / \mathrm{dL}$ em $3,4 \%$ das mulheres $(n=9)$. No que diz respeito à diferença média entre o hematócrito pré e pós-operatório foi $4,9 \pm 2,6 \%$, tendo a perda máxima sido $13,7 \%$.

Ocorreram complicações intra-operatórias major em duas $(0,76 \%)$ doentes, nomeadamente um caso de paragem cardio-respiratória durante a indução anestésica, em doente com antecedentes de timoma, que reverteu após manobras de reanimação; e um caso de conversão para laparotomia por hemorragia uterina generalizada difícil de controlar, sem qualquer lesão vascular iatrogénica específica (o mesmo caso em que ocorreu diminuição de $4,7 \mathrm{~g} / \mathrm{dL}$ de hemoglobina; trata-se de doente sem antecedentes de distúrbios da coagulação conhecidos) (Tabela 5). Salientam-se como complicações intra-operatórias minor hemorragia sem necessidade de transfusão sanguínea em nove doentes $(3,4 \%)$ e dois casos $(0,76 \%)$ de laceração iatrogénica da serosa do cólon sigmóide, com sutura imediata (Tabela 5).

Relativamente a complicações major no pós-operatório, ocorreu deiscência da cúpula vaginal em dois casos $(0,76 \%)$, um dos quais em mulher com adenocarcinoma do endométrio estádio IBG2 (a mesma doente submetida a linfadenectomia pélvica), após sessão de braquiterapia, e outro cinco meses após a cirurgia, na sequência de relações sexuais. Estas doentes foram submetidas a encerramento secundário da cúpula vaginal por via laparoscópica, após excisão do tecido fibrótico e necrosado dos bordos da cúpula, a fim de facilitar a cicatrização. No pós-operatório ocorreram situações infeciosas em 15 doentes $(5,7 \%)$, nomeadamente $11(4,2 \%)$ infeções do trato urinário inferior, dois $(0,76 \%)$ casos de febre, um $(0,38 \%)$ caso de infeção da cúpula vaginal e um $(0,38 \%)$ caso de infeção da cicatriz umbilical, com formação de fístula supra-aponevrótica, 
Tabela 5 - Complicações major e minor intra e pós-operatórias

\begin{tabular}{|c|c|c|}
\hline Complicações & $n$ & $\%$ \\
\hline \multicolumn{3}{|c|}{ Major } \\
\hline Deiscência da cúpula vaginal & 2 & 0,76 \\
\hline Complicações anestésicas major & 1 & 0,38 \\
\hline Conversão para laparotomia & 1 & 0,38 \\
\hline \multicolumn{3}{|c|}{ Minor } \\
\hline \multicolumn{3}{|l|}{ Infeciosas } \\
\hline Infeção do trato urinário inferior & 11 & 4,20 \\
\hline Febre (temperatura $>38^{\circ} \mathrm{C}$ nos primeiros 7 dias) & 2 & 0,76 \\
\hline Infeção da cúpula vaginal & 1 & 0,38 \\
\hline Infeção da cicatriz umbilical (com fístula) & 1 & 0,38 \\
\hline Hemorragia sem necessidade de transfusão sanguínea & 9 & 3,40 \\
\hline Incontinência urinária de esforço & 4 & 1,50 \\
\hline Lesão iatrogénica da serosa intestinal & 2 & 0,76 \\
\hline
\end{tabular}

que foi excisada três anos após a cirurgia. De realçar quatro $(1,5 \%)$ casos de incontinência urinária de esforço, dos quais dois persistiam 12 meses depois da HTL (Tabela 5). Após um ano de seguimento, não foi descrito qualquer caso de prolapso da cúpula vaginal. Não ocorreu nenhuma lesão ureteral ou vesical, nenhum reinternamento (menos de 72 horas após a alta), nem nenhum caso de morte intra-operatória ou no pós-operatório imediato. Assim, a morbilidade major global foi $1,5 \%(n=4)$ e a minor foi $11,5 \%$ $(n=30)$.

\section{DISCUSSÃO}

A histerectomia por via laparoscópica foi inicialmente alvo de críticas, devido à dificuldade técnica, maior tempo operatório e aparente aumento das complicações major, nomeadamente do trato urinário. Contudo, os seus benefícios incluem menos dor pós-operatória, menor tempo de internamento, recuperação mais rápida, com regresso mais precoce à atividade profissional, aumento da qualidade de vida a curto prazo e melhores resultados estéticos, comparativamente com a histerectomia abdominal. $8,9,15,21,22$ Do ponto de vista técnico, os benefícios da abordagem laparoscópica incluem a ampliação da imagem e visão de alta definição, facilitando o acesso aos vasos uterinos, ureter, reto e vagina. ${ }^{9}$ Apesar da controvérsia inicial relativa à taxa de complicações associada à via laparoscópica, os estudos mais recentes são consensuais ao apresentar a HTL como uma técnica segura e de primeira linha em múltiplas indicações cirúrgicas, quer benignas quer malignas..$^{9,10,13,14}$ Contudo, em Portugal, a histerectomia laparoscópica não teve ainda a implementação desejada. ${ }^{19}$

No presente estudo apresentamos a análise de 262 HTL realizadas por uma equipa cirúrgica num hospital do Serviço Nacional de Saúde. Os nossos resultados demonstram que, após o estabelecimento da técnica e treino da equipa, a via laparoscópica torna-se a abordagem preferencial para a realização de histerectomia. De facto, e de acordo com a literatura, após o primeiro ano do estudo, a via laparoscópica tornou-se a principal via de abordagem, com diminuição principalmente do número de histerectomias abdominais. ${ }^{3,9,23}$ De salientar, no entanto, que de acordo com uma revisão de 2009 da Cochrane, bem como as recomendações da American Congress of Obtetricians and Gynecologists (ACOG), dadas as suas vantagens e baixa taxa de complicações, a via vaginal se mantém a via de abordagem de primeira linha da histerectomia. A histerectomia laparoscópica deverá, assim, ser realizada sempre que a via vaginal não for exequível ou indicada, a fim de se evitar a via abdominal. ${ }^{6,8}$

Dado a histerectomia ser a cirurgia ginecológica major mais frequentemente realizada nos países desenvolvidos, num momento em que é premente a redução de custos no Sistema Nacional de Saúde, a avaliação do custo-eficácia das suas diferentes vias de abordagem deve, também, ser realizada. Toda a cirurgia laparoscópica exige equipamentos e instrumentos específicos e economicamente dispen- 
diosos. Estudos prévios demonstraram que a histerectomia por via laparoscópica era um procedimento mais dispendioso comparativamente com a abordagem abdominal. ${ }^{18,24}$ Contudo, nos últimos anos, com a maior experiência cirúrgica e, consequentemente, menor taxa de complicações, esta realidade económica tem-se alterado. A associação às vias de abordagem minimamente invasiva (vaginal e laparoscópica) de menor duração do internamento, menos dor pós-operatória, menor necessidade transfusional e taxa de complicações infeciosas, e regresso precoce à atividade profissional, tem permitido diminuir custos indiretos associados ao procedimento cirúrgico. Wright et al demonstraram, muito recentemente, que a histerectomia vaginal é o método menos dispendioso, seguida pela histerectomia laparoscópica. ${ }^{25}$ De facto, a via laparoscópica associou-se a menores custos comparativamente com a histerectomia abdominal. ${ }^{25,26}$ Exige-se, assim, que as supostas barreiras económicas impostas à implementação de histerectomia por via laparoscópica sejam ultrapassadas.

Das caraterísticas das doentes submetidas a HTL salienta-se que a maioria apresentava co-morbilidades médicas de relevo e IMC médio de $26,5 \mathrm{~kg} / \mathrm{m}^{2}$ (superior ao IMC correspondente a peso ideal de $20-24,9 \mathrm{~kg} / \mathrm{m}^{2}$ ), sendo que $42 \%$ das mulheres tinham excesso de peso ou eram obesas. Para além disso, quase metade das doentes $(49,2 \%)$ tinha pelo menos uma cirurgia abdomino-pélvica prévia.

As principais indicações cirúrgicas para a realização de histerectomia neste estudo estão de acordo com o descrito na literatura, assumindo a fibromiomatose uterina um papel preponderante $(53,4 \%) .{ }^{10,18,21,27,28}$ Por sua vez, os achados anatomo-patológicos estão de acordo com as principais indicações cirúrgicas, salientando-se a presença de fibroleiomiomas em $69,1 \%$ das peças operatórias.

Foram realizados outros procedimentos acessórios, com grau de complexidade variável, em 32,4\% das mulheres. Tal demonstra a eficácia da abordagem laparoscópica para diferentes procedimentos cirúrgicos necessários no âmbito da ginecologia, sem necessidade de conversão ou de um segundo tempo cirúrgico.

Nas nossas 262 HTL o tempo operatório médio foi 77,7 minutos, observando-se uma diminuição significativa do primeiro para o quarto ano do estudo. Estes valores estão de acordo com os descritos por grupos com experiência globalmente reconhecida, tal como Wattiez et al e Malzoni et al. ${ }^{15,16} \mathrm{O}$ grupo de Malzoni, por exemplo, descreveu um tempo cirúrgico médio de 80 minutos. ${ }^{16} \mathrm{O}$ tempo operatório aqui descrito é significativamente inferior ao descrito por Santos et al, numa revisão de 89 histerectomias vaginais assistidas por laparoscopia realizadas num serviço de ginecologia português, em que é apresentado um tempo médio de 120,6 minutos. ${ }^{18}$ Estes resultados demonstram que, perante uma equipa treinada, a laparoscopia não se associa a prolongamento do tempo operatório. Para além disso reforçam, mais uma vez, a importância do treino e de uma curva de aprendizagem inicial, inerente aos procedimentos laparoscópicos.

De realçar que este tempo operatório foi possível, mes- mo com úteros com peso similar ou superior ao descrito na literatura. ${ }^{9,21,29}$ Neste trabalho o peso médio da peça operatória foi $241 \mathrm{~g}$ (mínimo 34; máximo 1000). Donnez et al, numa revisão de 3190 histerectomias laparoscópicas, descreveram um peso médio de $230 \mathrm{~g} .{ }^{9}$ Por sua vez, o estudo de Santos et al refere um peso médio de $138,4 \mathrm{~g},{ }^{18}$ inferior ao descrito no presente trabalho. Estes resultados reforçam, assim, que a via laparoscópica é possível mesmo perante úteros volumosos.

A duração média do internamento pós-operatório foi $1,49 \pm 0,9$ dias, estando de acordo com o descrito na literatura internacional. Malzoni et al, por exemplo, num estudo envolvendo 396 e 624 mulheres, descreveu um tempo de internamento após a cirurgia de 2,4 e 2,3 dias, respetivamente. ${ }^{16}$ De referir um caso com duração de internamento pós-operatório de oito dias, relativo a uma doente com doença de von Willebrand de tipo 3, cujo internamento foi mantido para vigilância da hemostase e reposição do fator VII.

No que diz respeito à evolução pós-operatória, encontramos uma diminuição média da hemoglobina de $1,5 \mathrm{~g} / \mathrm{dL}$. Estes resultados são similares aos descritos por Malzoni et al que descrevem uma diminuição da hemoglobina no pós-operatório de 1,44 e 1,39 g/dL. ${ }^{16}$

Na presente análise de 262 HTL descrevemos uma taxa complicações major de $1,5 \%$ e de complicações minor de $11,5 \%$, não tendo ocorrido nenhum caso de lesão ureteral, vesical, perfuração intestinal ou morte. Esta taxa de complicações é comparável com as descritas na literatura. Numa série de 1120 histerectomias laparoscópicas, Karaman et al descreveram uma taxa de complicações major de $1 \%{ }^{13}$ Brummer et al realizaram um estudo retrospetivo multicêntrico na Finlândia, englobando 27.827 HTL, no qual a incidência de complicações major variou de 1,8\% entre 1992 e 1999 e $1 \%$ entre 2000 e $2005 .{ }^{23}$ Por sua vez, Donnez e Donnez no seu estudo prospetivo de 400 histerectomias laparoscópicas descreveram uma taxa de complicações major precisamente de $1,5 \% .{ }^{10}$ No que diz respeito às complicações minor, de realçar que consideramos como complicação minor a ocorrência de laceração iatrogénica da serosa do cólon sigmóide, com sutura imediata, o que para alguns autores deverá ser considerado uma intercorrência normal. ${ }^{15}$ Para além disso, consideramos também como morbilidade os casos de incontinência urinária, de acordo com o defendido por Walsh et al, em que a disfunção vesical deve ser avaliada como complicação a longo prazo da abordagem laparoscópica. ${ }^{4}$ Contudo, os estudos prévios de avaliação da taxa de complicações não incluem este parâmetro. Independentemente deste facto, a taxa de complicações minor aqui descrita é aceitável e está de acordo com a literatura atual. Por exemplo, Hoffman et al, na sua série de 108 HTL descrevem uma taxa de $17,6 \%$ de complicações minor. ${ }^{30}$

Relativamente a limitações do presente trabalho, podemos apontar a metodologia adotada. Em primeiro lugar, todos os dados foram obtidos através da consulta de processos clínicos, o que está dependente do registo claro e 
completo da informação. Por outro lado, tratou-se de um estudo observacional retrospetivo, sem grupo controlo. A realização de um estudo prospectivo com grupo controlo (via vaginal ou via abdominal) seria de grande utilidade clínica.

\section{CONCLUSÕES}

O presente estudo demonstra que a HTL é minimamente invasiva, associada a baixa taxa de complicações intra e pós-operatórias, mesmo em mulheres com elevado IMC e antecedentes de cirurgia abdomino-pélvica. De acordo com o nosso conhecimento, esta é a primeira série portuguesa

\section{REFERÊNCIAS}

1. Farquhar $\mathrm{CM}$, Steiner CA. Hysterectomy rates in the United States 1990-1997. Obstet Gynecol. 2002;99:229-34.

2. Canadian Institute for Health Information. Health Care in Canada 2010. Ottawa: CIHI; 2010.

3. Wright JD, Ananth CV, Lewin SN, Burke WM, Lu YS, Neugut AI, et al. Robotically assisted vs laparoscopic hysterectomy among women with benign gynecologic disease. JAMA. 2013;309:689-98.

4. Walsh CA, Walsh SR, Tang TY, Slack M. Total abdominal hysterectomy versus total laparoscopic hysterectomy for benign disease: a meta-analysis. Eur J Obstet Gynecol Reprod Biol. 2009;144:3-7.

5. Whiteman MK, Hillis SD, Jamieson DJ, Morrow B, Podgornik MN, Brett $\mathrm{KM}$, et al. Inpatient hysterectomy surveillance in the United States, 2000-2004. Am J Obstet Gynecol. 2008;198:34:e1-7.

6. ACOG. ACOG Committee Opinion No. 444: choosing the route of hysterectomy for benign disease. Obstet Gynecol. 2009;114:1156-8.

7. Reich H, DeCaprio J, McGlynn F. Laparoscopic hysterectomy. J Gynecol Surg. 1989;5:213-6.

8. Nieboer TE, Johnson N, Lethaby A, Tavender E, Curr E, Garry R, et al. Surgical approach to hysterectomy for benign gynaecological disease. Cochrane Database Syst Rev. 2009;3:CD003677.

9. Donnez O, Jadoul P, Squifflet J, Donnez J. A series of 3190 laparoscopic hysterectomies for benign disease from 1990 to 2006: evaluation of complications compared with vaginal and abdominal procedures. BJOG. 2009;116:492-500.

10. Donnez O, Donnez J. A series of 400 laparoscopic hysterectomies for benign disease: a single centre, single surgeon prospective study of complications confirming previous retrospective study. BJOG. 2010;117:752-5

11. Garry R, Fountain J, Mason S, Hawe J, Napp V, Abbott J, et al. The eVALuate study: two parallel randomised trials, one comparing laparoscopic with abdominal hysterectomy, the other comparing laparoscopic with vaginal hysterectomy. BMJ. 2004;328:129.

12. Johnson N, Barlow D, Lethaby A, Tavender E, Curr E, Garry R. Surgical approach to hysterectomy for benign gynaecological disease. Cochrane Database Syst Rev. 2006;2:CD003677.

13. Karaman Y, Bingol B, Günenç Z. Prevention of complications in laparoscopic hysterectomy: experience with 1120 cases performed by a single surgeon. J Minim Invasive Gynecol. 2007;14:78-84.

14. Bojahr B, Raatz D, Schonleber G, Abri C, Ohlinger R. Perioperative complication rate in 1706 patients after a standardized laparoscopic supracervical hysterectomy technique. J Minim Invasive Gynecol. 2006;13:183-9.

15. Wattiez A, Soriano D, Cohen SB, Nervo P, Canis M, Botchorishvili R, et al. The learning curve of total laparoscopic hysterectomy: comparative analysis of 1647 cases. J Am Assoc Gynecol Laparosc. 2002;9:339-45.

16. Malzoni M, Perniola G, Perniola F, Imperato F. Optimizing the total laparoscopic hysterectomy procedure for benign uterine pathology. J Am As- de HTL e reforça que, se realizada por uma equipa cirúrgica adequadamente treinada, a HTL é segura, pode ser realizada num tempo operatório aceitável e é possível em úteros volumosos.

\section{CONFLITOS DE INTERESSE}

Os autores declaram a inexistência de conflitos de interesse na realização do presente trabalho.

\section{FONTES DE FINANCIAMENTO}

Não existiram fontes externas de financiamento para a realização deste artigo.

soc Gynecol Laparosc. 2004;11:211-8.

17. SOGC clinical guidelines. Hysterectomy. J Obstet Gynaecol Can. 2002;24:37-61

18. Santos E, Dias I, Varela MF, Freire-de-Oliveira C. Morbidity in laparoscopically assisted vaginal hysterectomies. Acta Obstet Ginecol Port. 2007;1:66-73.

19. Ordem dos Médicos. Um estudo da situação da laparoscopia, em Ginecologia, no país (2011); [consultado 2013 Mar 23]. Disponível em: https://www.ordemdosmedicos.pt/?lop=conteudo\&op=8eefcfdf5990e44 1 fOfb6f3fad709e21\&id=64c31821603ab476a318839606743bd6.

20. World Health Organization. Obesity: preventing and managing the global epidemic. Report on a WHO Consultation on Obesity, 3-5 June 1997, Geneva, WHO/NUT/NCD/98.1. Technical Report Series Number 894 Geneva: WHO; 2000

21. Cho HY, Choi KJ, Lee YL, Chang KH, Kim HB, Park SH. Comparison of two bipolar systems in laparoscopic hysterectomy. JSLS. 2012;16:45660 .

22. Kluivers KB, Hendriks JC, Mol BW, Bongers MY, Bremer GL, de Vet HC et al. Quality of life and surgical outcome after total laparoscopic hysterectomy versus total abdominal hysterectomy for benign disease: a randomized, controlled trial. J Minim Invasive Gynecol. 2007;14:145-52.

23. Brummer TH, Seppälä TT, Härkki PS. National learning curve for laparoscopic hysterectomy and trends in hysterectomy in Finland 2000-2005. Hum Reprod. 2008;23:840-5.

24. Bijen CB, Vermeulen KM, Mourits MJ, de Bock GH. Costs and effects of abdominal versus laparoscopic hysterectomy: systematic review of controlled trials. PLoS One. 2009;4:e7340.

25. Wright KN, Jonsdottir GM, Jorgensen S, Shah N, Einarsson Jl. Costs and outcomes of abdominal, vaginal, laparoscopic and robotic hysterectomies. JSLS. 2012;16:519-24

26. Cho HY, Park ST, Kim HB, Kang SW, Park SH.Surgical Outcome and Cost Comparison Between Total Vaginal Hysterectomy and Laparoscopic Hysterectomy for Uteri Weighing > $500 \mathrm{~g}$. J Minim Invasive Gynecol. 2014;21:115-9.

27. Broder MS, Kanouse DE, Mittman BS, Bernstein SJ. The appropriateness of recommendations for hysterectomy. Obstet Gynecol. 2000;95:199-205.

28. Perino A, Cucinella G, Venezia R, Castelli A, Cittadini E. Total laparoscopic hysterectomy versus total abdominal hysterectomy: an assessment of the learning curve in a prospective randomized study. Hum $\mathrm{Re}-$ prod. 1999;14:2996-9.

29. Paraiso MF, Ridgeway B, Park AJ, Jelovsek JE, Barber MD, Falcone T, at al. A randomized trial comparing conventional and robotically assisted total laparoscopic hysterectomy. Am J Obstet Gynecol. 2013;208:e1-7.

30. Hoffman CP, Kennedy J, Borschel L, Burchette R, Kidd A. Laparoscopic hysterectomy: the Kaiser Permanente San Diego experience. J Minim Invasive Gynecol. 2005;12:16-24. 


\section{Histerectomia Totalmente Laparoscópica: Análise Retrospetiva de 262 Casos

\author{
Acta Med Port 2014:27:73-81
}

Publicado pela Acta Médica Portuguesa, a Revista Científica da Ordem dos Médicos

Av. Almirante Gago Coutinho, 151

1749-084 Lisboa, Portugal.

Tel: +351 218428215

E-mail: submissao@actamedicaportuguesa.com

www.actamedicaportuguesa.com

ISSN:0870-399X | e-ISSN: 1646-0758

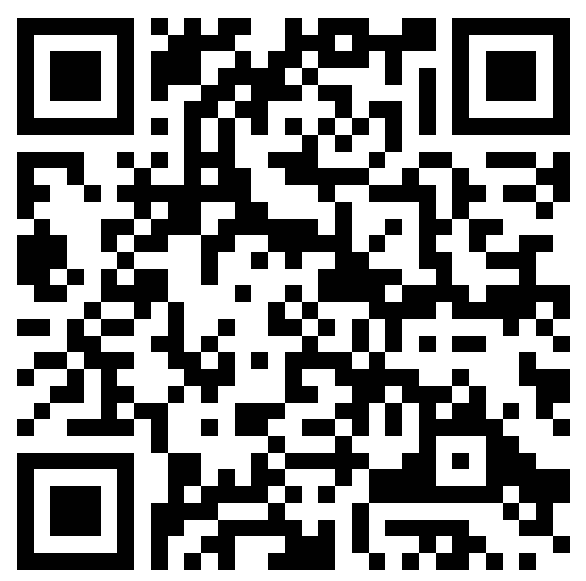

